\title{
Liquid marbles: Physics and applications
}

\author{
NACHIKETA JANARDAN ${ }^{1}$, MAHESH V PANCHAGNULA ${ }^{1, *}$ \\ and EDWARD BORMASHENKO ${ }^{2}$
}

\author{
${ }^{1}$ Indian Institute of Technology Madras, Chennai, 600036, India \\ ${ }^{2}$ Physics Faculty, Ariel University, Ariel 40700, Israel \\ e-mail: mvp@iitm.ac.in
}

MS received 18 July 2014; revised 8 October 2014;accepted 7 November 2014

\begin{abstract}
Liquid marbles are formed by encapsulating microscale volume of liquid in a particulate sheath. The marble thus formed is robust and resists rupture if the particulate layer covers the entire volume of liquid and prevents contact between the liquid and the substrate. Liquid marbles have been objects of study over the past decade. Research has been focused on understanding their formation and properties - both static and dynamic. A range of particulate materials as well as liquids have been employed to make these objects. This paper summarizes the state of the art in this regard and discusses new developments that are being discussed. Finally, some directions are proposed based on lacunae observed in the community's understanding - both in terms of the science as well as on the application front.
\end{abstract}

Keywords. Liquid marbles; wetting; hydrophobic particles; Cassie-Baxter; superhydrophobic.

\section{Introduction}

Advances in surface science have led to an explosion of interest in the areas of hydrophobicity and superhydrophobicity. Superhydrophobic surfaces can be created by the process of surface roughening and etching, to create surfaces with a variety of properties. However, all these methods involve modification of the surface. Liquid marbles offer an alternative method to generate superhydrophobic behavior without modifying the surface. These liquid marbles were first reported in the work of Mahadevan \& Pomeau (1999) and Aussillous \& Quéré (2001). Since then, they have been the focus of intense study over the past decade. Figure 1 is a graph depicting the number of publications and citations outside the author set publishing in this area of research. As can be seen, interest in this topic is rapidly increasing both within the liquid marbles community as well as from the outside in the form of envisaged applications being researched.

Liquid marbles are entities formed from liquids and particles interacting. The particles assemble on the interface and encapsulate the liquid inside, thereby preventing direct contact between the surface and the liquid. The resulting "liquid marble" (see figure 2) acts like a drop on a 


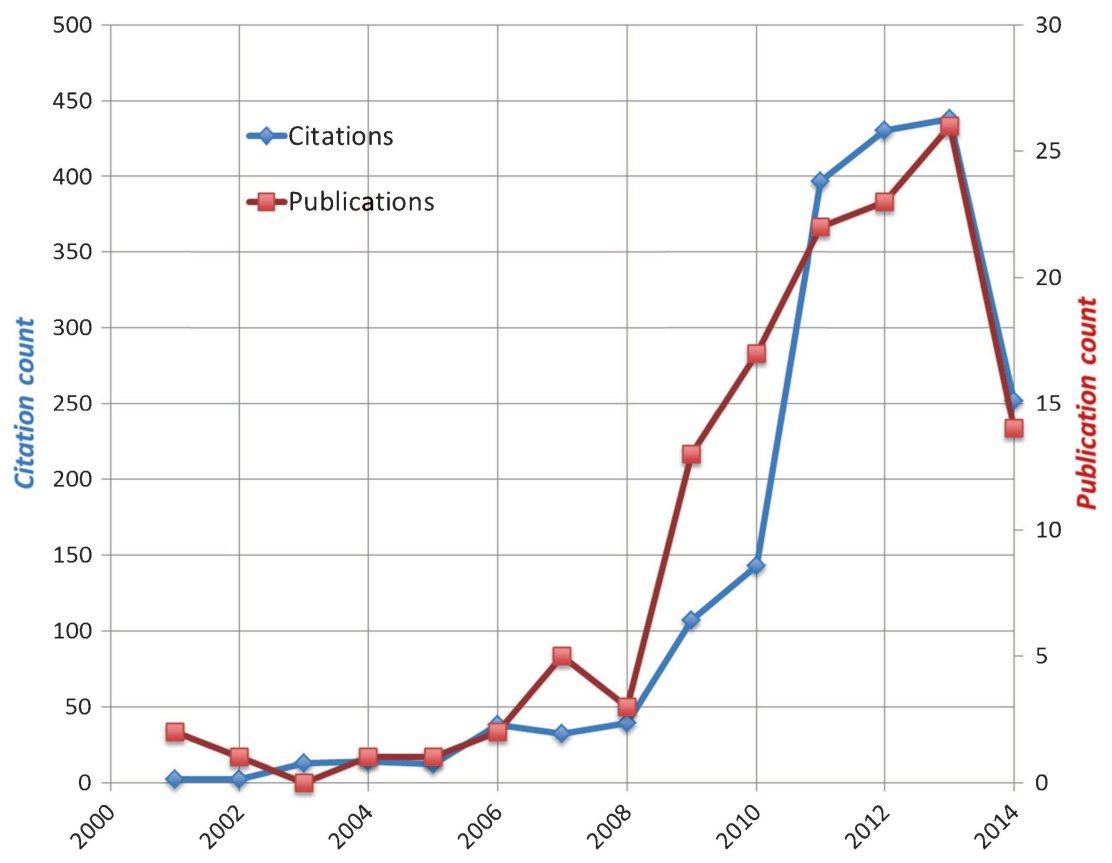

Figure 1. Journal publications and non-co-author citations for the keyword "liquid marbles". Data obtained from Scopus ${ }^{\circledR}$.

superhydrophobic surface and can have a high apparent contact angle and extremely low friction while rolling on surfaces. Liquid marbles have great potential in a wide range of applications from water quality testing as shown by Bormashenko \& Musin (2009) to manipulation and transport of minute quantities of liquid in microfluidic system as demonstrated by Venkateswara Rao

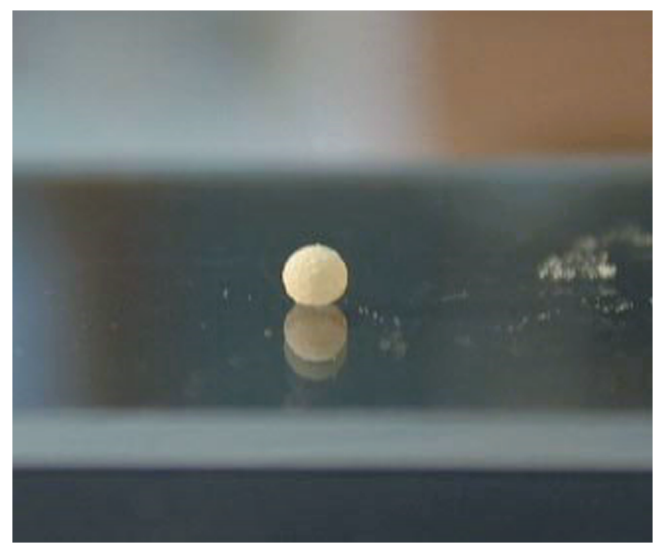

Figure 2. A liquid marble. Source: Aussillous \& Quéré (2001). Reprinted by permission from Macmillan Publishers Ltd: Nature, (Aussillous P and Quéré D 2001 Liquid marbles. Nature 411: 924-927), copyright (2001). 
et al (2005). In addition, a number of other applications are being investigated. The possibility of actuating liquid marbles using electric and magnetic stimuli promises other exciting new applications in the future. These applications will be discussed later.

Liquid marbles are typically made by coating several microliters volume of a liquid in a matrix of hydrophobic particles. More recently, Dandan \& Erbil (2009) have demonstrated that it is also possible to use hydrophilic particles to produce these marbles. In either case, the surface energy of the liquid marble is considerably less than the individual surface energies of the liquid and the particles. Thus, regardless of the intrinsic contact angle of the liquid on the given substrate, the effective surface energy is reduced in the process of the formation of liquid marbles. Some examples of hydrophobic particles that have been used to manufacture liquid marbles are polytetrafluoroethylene (PTFE), hydroxydimethylsilicone (HDMS), $\mathrm{Fe}_{3} \mathrm{O}_{4}$, fumed silica nanoparticles as well as other polymers. Hydrophilic particles that have been used include graphene and carbon black. Recently, Mele et al (2014) employed nanofibres to manufacture liquid marbles. These have been shown to possess better structural properties than marbles made from particulate matter. Similarly, different liquids have been used to make liquid marbles. Most of these liquids are known to be high surface tension liquids, with surface tension values ranging from 40 to $70 \mathrm{~mJ} / \mathrm{m}^{2}$. This requirement is important because the effective surface tension, which holds the marble intact, is strongly dependent on the inherent liquid-vapor interfacial tension. The lower this value, the less robust (in general) such a marble may be.

Liquid marbles have been shown to remain stable due to a pocket of air trapped stably between the liquid and the substrate. This observation has arisen out of research from several reports such as that of Biance et al (2003), Nosonovsky \& Bhushan (2008), Nosonovsky \& Bormashenko (2009), and Quéré \& Reyssat (2008). In areas where the colloidal particles do not cover the liquid completely, the liquid does not rest on a cushion of air. Such liquid marbles are susceptible to rupture. The existence of this air layer was also experimentally confirmed by Bormashenko et al (2009a). They performed an elegant experiment in which a marble of liquid $\mathrm{NaOH}$ was allowed to come in contact with a substrate containing phenolphthalein. The $\mathrm{NaOH}$ marble failed to change the color of the phenolphthalein sheet thus confirming that the liquid was not directly in contact with the surface. Because there is no direct interaction between the liquid and the surface, the effective surface tension of these liquid marbles will differ from the surface tension of the pure liquid. Liquid marbles are even able to float on liquids without mixing with them as shown by Bormashenko et al (2009a). They merely cause a depression on the liquid surface. In other instances, the liquid marbles have even been shown to sink into the liquid without losing cohesion or disintegrating as demonstrated by Mele et al (2014). Figure 3 is a picture of such an experiment in which a liquid marble is allowed to sink into water without disintegrating resulting in a Pickering emulsion. Such properties of liquid marbles can be exploited in applications where controlled release is required, such as in drug delivery.

The sheath of the colloidal particles on the surface of the liquid is in general non-uniform and thus the space between the particles on the interface is open to penetration by gas as can be seen in figure 4 (Aussillous \& Quéré 2001). This property of a liquid marble was exploited for gas sensing by Arbatan \& Shen (2010). The evaporation of the liquid phase upon heating from the liquid marble has also been studied extensively by Dandan \& Erbil (2009) and Tosun \& Erbil (2009). Figure 5 shows the evaporation of liquid from a graphite-water marble. It can be seen that the process leaves behind a dehydrated shell of graphite. Therefore, designing the porosity of the sheath can control the transport of liquid from the liquid marble. The porosity in turn can be controlled by choice of the encapsulating material. In summary, liquid marbles hold potential for several applications owing to their versatile physical and chemical characteristics. 
(a)
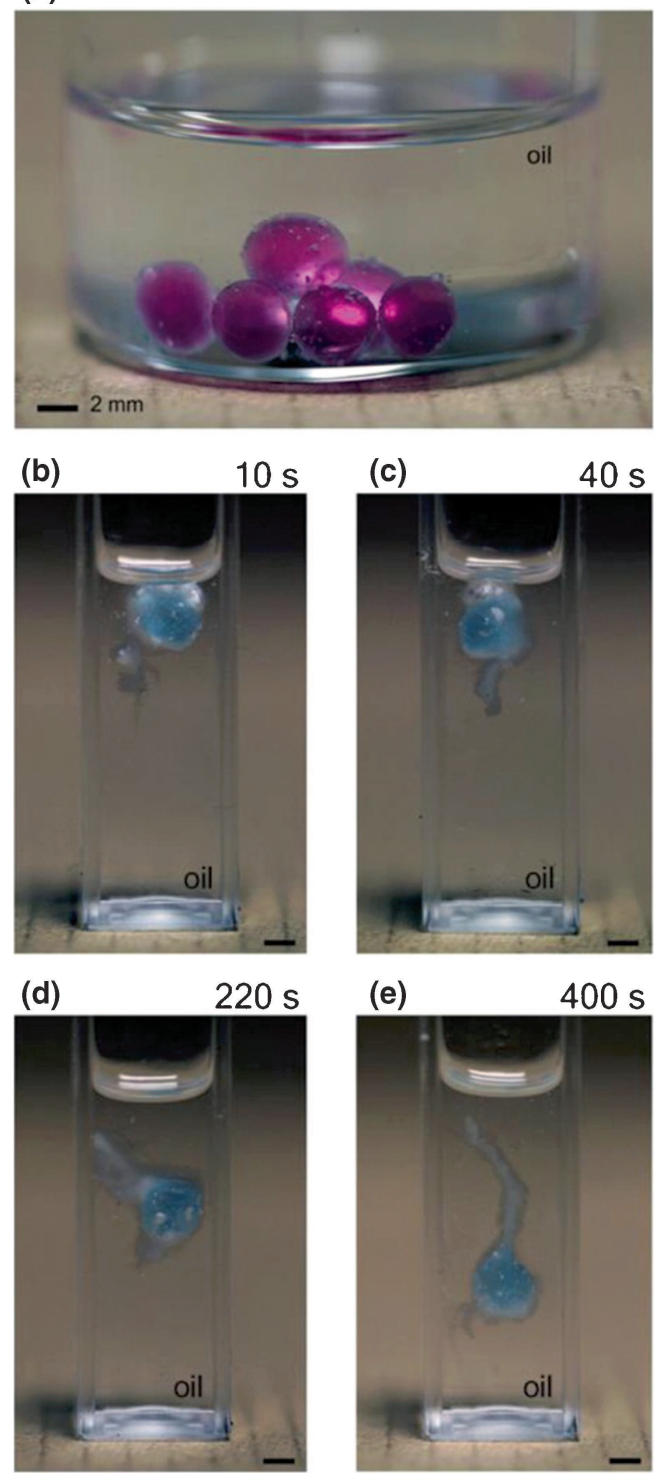

(e)

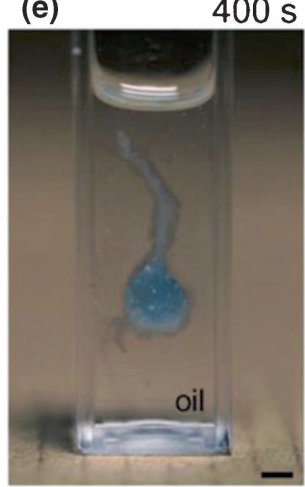

Figure 3. Liquid marbles in water. Source: Mele et al (2014). Reprinted (adapted) with permission from Mele E, Bayer I S, Nanni G, Heredia-Guerrero J A, Ruffilli R, Ayadi F, Marini L, Cingolani R and Athanassiou A 2014 Biomimetic approach for liquid encapsulation with nanofibrillar cloaks. Langmuir 30: 2896-579 2902. Copyright (2014), American Chemical Society.

Nguyen et al (2010) performed many studies upon the methodology of the manufacture of liquid marbles and the energy transport associated with it. Comparisons were made between marbles manufactured in the traditional rolling process and those manufactured by the dropping of water droplets onto a bed of superhydrophobic particles. The marbles manufactured in the latter method were shown to have non-uniform coverage of particles on the liquid-vapor interface. Indeed, the uppermost area of the marble was left uncovered. This has a bearing on their robustness. 


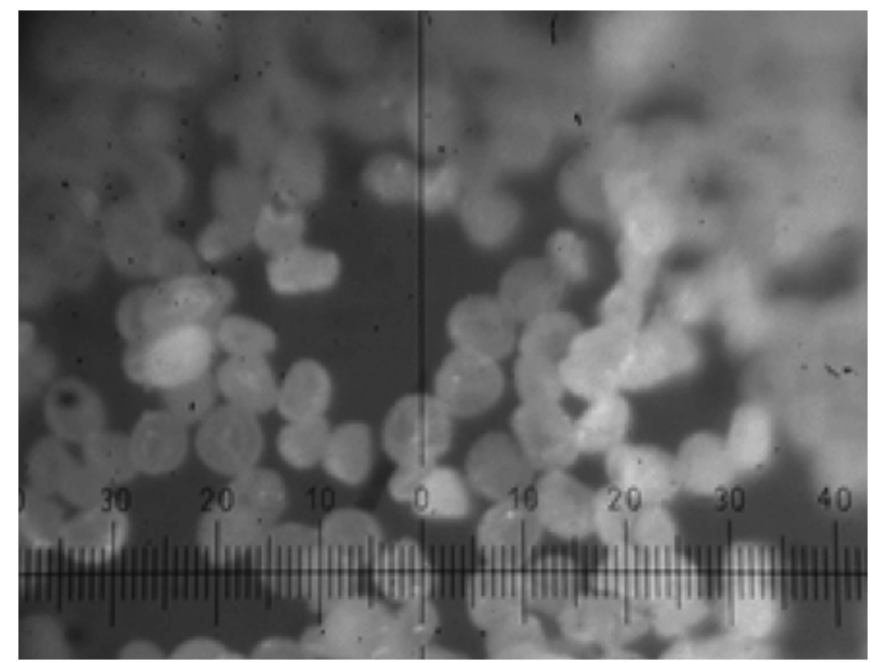

Figure 4. Surface of the liquid marble with lycopodium grains on the surface. Source: Aussillous \& Quére (2006). Aussillous \& Quere, Properties of Liquid Marbles, Proceedings of the Royal Society A, 2006, 462, 2067, by permission of the Royal Society.

\section{Effective surface tension of liquid marbles}

The effective surface tension of liquid marbles - its estimation and measurement - is a subject that has not received much consideration in the literature. This parameter is important, however, because it holds the key to determining the rupture strength and robustness of liquid marbles. Experimental methods such as the measurement of the height of the liquid marble, analysis of the shape, and vibration of marbles have been utilized to estimate the effective surface tension

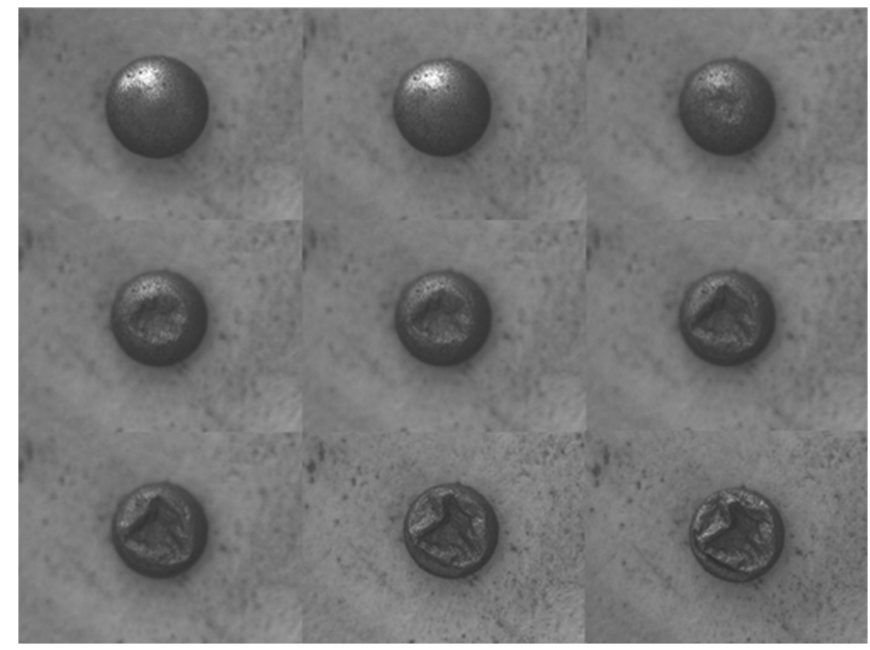

Figure 5. Evaporation of Graphite covered liquid marbles. Source: Dandan \& Erbil (2009). Reprinted (adapted) with permission from Dandan M and Erbil H Y 2009 Evaporation rate of graphite liquid marbles: comparison with water droplets. Langmuir 25: 8362-8367. Copyright (2009) American Chemical Society. 
of the liquid marble in many works such as Bormashenko et al (2013, 2009b, 2009c). We will discuss a few methods that have been proposed to measure this parameter.

\subsection{The puddle height method}

The puddle height method is perhaps the simplest method used to estimate the effective surface tension of the marble. At small volumes, a liquid marble can be approximated to be a sphere, but as the volume increases it takes on the shape of a puddle. This was demonstrated by Aussillous \& Quere $(2001,2006)$ and Bormashenko et al (2009b). It can be shown that the effective surface tension $\gamma_{\mathrm{eff}}$ in this case can be written as

$$
\gamma_{\mathrm{eff}}=\frac{\rho g H^{2}}{4}
$$

where $\rho$ is the density of the liquid and $H$ is the maximum height of the marble.

\subsection{Analysis of the marble shape}

An extension of the previously discussed method is to take the complete marble shape into consideration. In this approach, an analysis of the shape of the liquid marbles can be used to calculate the effective surface tension. However, it must be noted that an accurate description of the shape can only be numerically determined. When a liquid marble is deformed by gravity, the shape can be described (for low Bond numbers or small effect of gravity) as that of an oblate spheroid as shown by Whyman \& Bormashenko (2009). The effective surface tension of the liquid marble is determined by of the fitting an ellipse and relating the effective surface tension to the measured geometrical parameters.

\subsection{Vibration method}

The measurement of the resonance frequencies of liquid marbles can be used to determine the effective surface tension of the liquid marbles. This approach was suggested by Bormashenko et al (2009c) and Celestini \& Kofman (2006). In this approach, the liquid marble is placed on a vibration platform and oscillated in the direction of gravity. The resonant frequency at which the deformation reaches a maximum is measured directly. The effective surface tension can be calculated from this measurement as

$$
\gamma_{\mathrm{eff}}=\frac{2 \pi \rho V f^{2}}{h(\theta)(1-\cos \theta)},
$$

where $f, V$, and $\theta$ are the resonant frequency, volume and the contact angle of the liquid marble respectively. $h(\theta)$ is a numerical multiplier whose value can be found in Celestini \& Kofman (2006).

The effective surface tension of the liquid marble is dependent on the type of particles that are used to make the liquid marble. All the three methods described above have their own advantages and disadvantages. The puddle height method, being the simplest to employ, can perhaps be recommended in general. It has been shown that the effective surface tension can be greater or lesser than the surface tension of the pure liquid depending on the nature of the force between the particles and the liquid. The high scattering in experimental measurements of the effective 
Table 1. Values of surface tension measured with different methods.

\begin{tabular}{lccc}
\hline Liquid marbles & \multicolumn{3}{c}{$\gamma, \mathrm{mJ} / \mathrm{m}^{2}$} \\
\cline { 2 - 4 } Particulate material & Maximal height method & Marble shape analysis method & Vibration method \\
\hline PVDF & $70 \pm 7$ & $79 \pm 5$ & $75 \pm 3$ \\
PE & $66 \pm 5$ & $63 \pm 3$ & $60 \pm 4$ \\
Teflon & $60 \pm 6$ & $53 \pm 5$ & $53 \pm 3$ \\
Lycopodium & $50 \pm 5$ & $62 \pm 5$ & $43 \pm 3$ \\
Carbon black & $66 \pm 4$ & $68 \pm 3$ & - \\
\hline
\end{tabular}

surface tensions with various techniques should be emphasized and requires further study (see table 1).

The ambiguity of the notion of the effective tension of liquid marbles may be rationalized if we consider its pronounced hysteretic nature as demonstrated by Bormashenko et al (2013). Marbles made from the same liquid and particulate material (and of the same volume) show greatly varying effective surface tension depending on whether the particles assembled from a dry (from outside) or wet (from inside) state. In addition, the experiments performed with inflated and deflated liquid marbles demonstrated that the effective surface tension (calculated from the marbles' shapes) depended on the volume of a marble and varies widely. In their study, three "surfaces phases" are distinguished within the inflation-deflation cycles. They are (i) a dilute distribution of solid particles on the surface of the marble, (ii) a uniform distribution of solid particles on the surface of the liquid marble and (iii) a stage corresponding to the buckling of the solid coating which was observed by Monteux et al (2007) and Bhosale \& Panchagnula (2010). The effective surface tension of the marble has been shown to depend on the surface phase associated with the particulate matter. Therefore, ascribing a unique value to the effective surface tension of liquid marbles for a given pair of liquid and particulate material is erroneous and needs to be avoided.

\section{Elastic properties of liquid marbles}

Liquid marbles show interesting elastic properties. Their surfaces demonstrate not only innate liquid-like but also soft elastic solid-like properties. For example, liquid marbles do not coalesce when they collide unless an energy barrier required for rupture is overcome. Vella et al (2004) developed a model for predicting the elastic properties of liquid surfaces coated by identical solid particles with the diameter of $d$. These surfaces behave like two-dimensional elastic solids and can support anisotropic stresses and strains. In the simplest case, however, they may be characterized using an effective Young's modulus. They proposed a model for the effective Young's modulus of the "interfacial particle raft" in the form of

$$
E=\frac{1-v \gamma}{1+\phi d}
$$

where $v$ is the Poisson ratio of solid particles, and $\phi$ is the solid fraction of the interface. They concluded that the elastic properties of such an interface are not dependent on the capillary interaction between particles; however, this hypothesis needs experimental verification. Lack of experimental data relating to the elastic properties of self-assembled particle systems on liquid interfaces is noteworthy and beckons investigation. 


\section{Other physical properties}

The density, viscosity and elastic properties of liquid marbles are other interesting properties of these systems and have been studied during the past decade. It has been shown by many groups, for example, Aussillous \& Quéré (2001), Richard \& Quéré (1999) and Mahadevan \& Pomeau (1999), that a liquid marble does not slide down an incline; instead, it has been shown to manifest rolling motion. The dynamics of such a liquid marble in motion is determined by the dimensionless Capillary number, $C a=\frac{\eta v}{\gamma}$ where $\eta$ is the viscosity, $v$ is the characteristic velocity and $\gamma$ is the surface tension and other dimensionless groups such as the Reynolds number $(R e)$

The static shape of the liquid marble is determined by the interplay of surface tension forces and the gravitational force that is acting on the marble. When the characteristic length of the marble is of the order of the capillary length, the effects of gravity become comparable to the effects of the surface tension forces. The capillary length of a liquid marble is given by $l_{c}=$

$\sqrt{\frac{\gamma_{\text {eff }}}{\rho g}}$. The effect of gravity is to flatten the drop and increase the surface area that is in contact with the substrate. Increasing the volume of the drop leads to the formation of puddles instead of drops. Puddles by their nature are unstable and do not give rise to stable formations. In addition, the mobility is greatly hindered owing to higher rolling resistance. Thus the maximum volume of liquid that can be used to make a liquid marble is around $50 \mu \mathrm{L}$.

Thus the two characteristic lengths that are applicable are the radius of the drop (or a length scale based on the drop volume) and the radius of the circular contact area between the drop and the surface. In general, the ratio of these two length scales is a function of $l_{c}$ and $C a$.

It has been predicted analytically and subsequently verified experimentally by several groups such as de Gennes et al (2003) and Richard \& Quéré (1999) that smaller marbles descend down an inclined plane faster than heavier marbles. This counter-intuitive result is ascribed to the fact that the velocity of the center of mass of the marble scales inversely with the radius of the marble as

$$
v=\frac{\gamma l_{c}}{\eta R} \sin \alpha
$$

where $R$ is the radius. $\alpha$ is the inclination of the surface.

Liquid marbles are generally robust and can resist shocks and manipulation as was shown by Aussillous \& Quéré (2006). The marbles were able to resist being squeezed between two plates up to a maximum, beyond which the marbles ruptured. The surface area of the marbles increased when squeezed and from their calculations, it was seen that the maximum increase that was sustainable was around $25 \%$ of the original surface area. Similarly, dropping the liquid marbles from a height resulted in their destruction when dropped beyond a maximum height. The maximum height beyond which the drops ruptured depended on the viscosity and the uniformity of coverage of the solid particles.

\section{Manufacture of liquid marbles}

Liquid marbles are generally prepared by depositing a small volume of liquid on a hydrophobic nano/microparticulate powder. The liquid droplet is then rolled in the powder and the particles are allowed to self-assemble on the droplet. This process creates a layer of particles on the droplet interface that act as a shell and generate the properties that are associated with the liquid marble. However, this method is labor intensive and not conducive to the continuous manufacture of liquid marbles. Other methods such as proposed by Binks \& Murakami (2006) also do not present control over the size of the marbles and the distribution width. Another important limitation of 
the classical preparation methods is that the drop that can be made to detach from the syringe $(\sim 10 \mu \mathrm{L})$ limits the minimum size of the marble. On the other hand, smaller marbles have been shown to be more stable and hence a drop volume of less than $10 \mu \mathrm{L}$ is desirable. The inherent variability in the rolling process also does not ensure a uniform coverage of the hydrophobic particles on the liquid-air interface is not uniform. This uncertainty of manufacture and the associated property variability has been identified as one of the primary limitations in using liquid marbles in industrial scale applications.

To address this issue, Bhosale \& Panchagnula (2012) have recently reported a novel method by which liquid marbles can be manufactured by the process of condensation and drop nucleation. In their method, the liquid marbles were self-assembled using physical processes, instead of being manually crafted. The schematic for their experimental apparatus used is given in figure 6. A glass vial containing a liquid was set up on a heating plate. The liquid-air interface was covered with a layer of microparticulate matter that was a few hundred microns thick. The glass vial was filled with a small amount of desired liquid (glycerol, ethylene glycol, or water). The hydrophobic powder was spread in a thin layer on this surface. The powder used in their experiments was Cab-O-Sil ${ }^{\circledR}$ hydrophobic fumed silica and micron-sized Teflon ${ }^{\circledR}$ ( $\mu$ PTFE). The vial was then placed on the heating pad and heat was supplied from the bottom. A thermocouple was used to continuously monitor the liquid temperature and an optical microscope was set up to capture the entire process for imaging. After heating for a while, the vapor that is released at the liquid-air meniscus condensed on the hydrophilic defect sites on the particulate matter. The drops thus nucleated are enveloped by the particulate matter through a self-assembly process. Thus, a liquid 'micro-marble' is formed, since the drops are only a few microns in diameter at this stage. The marbles were then scooped off the surface and tested for robustness and size. The experiment was repeated over a wide range of temperatures. The rate of formation of the marbles and their size distributions were noted.

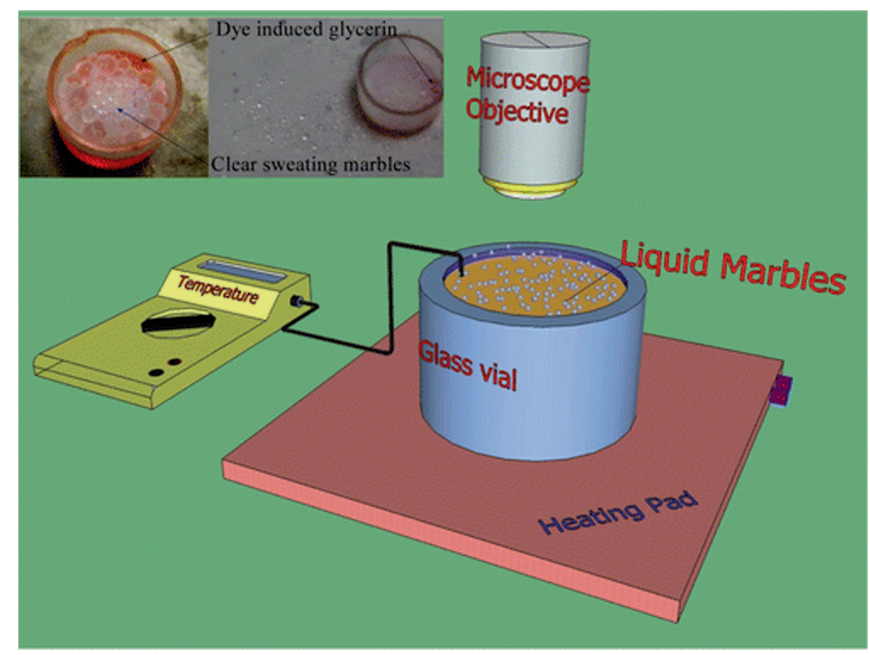

Figure 6. Schematic of the experimental set-up to produce sweating liquid marbles. Source: Bhosale \& Panchagnula (2012). Reprinted (adapted) with permission from Bhosale P S and Panchagnula M V 2012 Sweating liquid micro-marbles: dropwise condensation on hydrophobic nanoparticulate materials. Langmuir 28: 14860-. Copyright (2012) American Chemical Society. 
The initial focus of their study was the behavior of liquid marbles on heated surfaces. When the parent liquid marble was heated on a surface, it was noted that small liquid droplets started to appear at the interface of the marble and the air. These were small liquid droplets of self-encapsulated free particulate material and grew over time. When the heating process was continued, it was noticed that the daughter marble count and the size of the said daughter marbles both increased. Eventually, the daughter liquid marbles rolled down the surface of the parent marble. At some point, the parent marble was fully converted into daughter marbles. The appearance that the parent marbles appeared to sweat, led to the process being named as a "sweating" process. It was noted that the liquid marbles formed by this "sweating process" were more robust and were of nearly uniform size than the classical rolling process. The liquid marbles thus manufactured range in diameter from $3 \mu \mathrm{m}$ to $1000 \mu \mathrm{m}$. In addition, the particulate aggregates responsible for the marble production are also a few micrometers in size.

$\mu$ PTFE particles were initially used in the manufacture of such marbles. However, previous studies by Bhosale et al (2008) had shown that liquid marbles made from nanoparticles were more robust than those made from microparticles. Therefore, it was shown by Bhosale \& Panchagnula (2012) that using nanoparticulate fumed silica in the sweating process yields robust marbles with good control. In addition, by controlling the temperature and time of heating, they were able to gain control over the marble size distribution. This is the novelty in their process apart from the fact that this process lends itself to be converted into a continuous marble production system.

Analysis of their experimental results determined that three physical processes, viz. nucleation, condensation and coalescence, drove the production of the marbles. It was seen that initially, the marbles formed at seemingly random defect sites on the particulate material surface. Over time these marbles grew and started to coalesce forming bigger marbles, while new sites of marble nucleation were also being set up. This process is similar to the condensation and growth of droplets on superhydrophobic surfaces as studied by Beysens (2006). The nanoparticulate matrix is infused with vapor from the liquid as a result of the heating. The particulate matrix is made up of long chains of nanoscale fumed silica that has been silanized. From this process, there remain a few sites, which are hydrophilic. These hydrophilic spots on otherwise hydrophobic material provide the nucleation site for the condensed liquid. Once nucleation happens, the water vapor around the particles condenses preferentially on the water droplets at these sites, resulting in the continuous growth of these droplets. Many such nucleation sites form in the particulate matrix, as this system is quasi three-dimensional. These nucleated drops then merge and coalesce. The nucleated drops continue to grow until a threshold size is reached. Throughout this process, the hydrophobic particles around the drop deposit on the surface of the drop, thus forming a liquid marble. A schematic of this evaporation, nucleation and condensation process has been given in figure 7. The marbles are then removed from the particle surface. The size of the marbles can be controlled by controlling the rate of heating of the liquid. This process is remarkably simple and represents a major advance in the manufacture of such marbles, especially as an enabler for industrial applications. A photograph of the "sweating liquid marble" is given in figure 8 .

The rate of growth in the above-discussed system is observed to be a function of the temperature at which these marbles are produced. This is explained by the fact that the higher the heating temperatures, the faster the evaporation. This also results in the faster growth of the liquid marbles, a higher mean size of the liquid marble and a greater distribution width. Energetic barriers to coalescence are also lowered by the rise in temperature. Finally, coalescence is a strong function of the spatial proximity of the two daughter marbles. These processes tend to destroy the monodispersity, which is a feature of the nucleation/growth phase. 


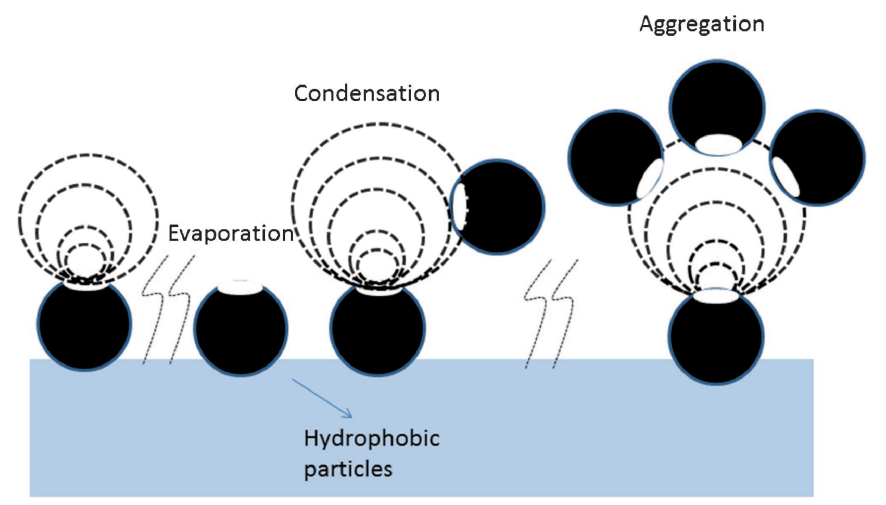

Figure 7. Schematic of the evaporation condensation process.

The overall efficiency of the process of conversion of the bulk of the liquid into the marbles can be quantitatively estimated in terms of the mass of liquid converted into daughter marbles at a given temperature and heating time. The mass conversion efficiency was measured to be as high as $50 \%$ at $220^{\circ} \mathrm{C}$ within a time span of two hours. Extremely small liquid marbles with diameters as small as $3 \mu \mathrm{m}$ (and with a narrow size distribution) can also be created by using low heating temperatures over smaller time intervals. Continuous production of liquid marbles would involve heating at low temperatures for an extended interval of time followed by a continuous removal of daughter marbles from the surface. The removal can be achieved through either mechanical or pneumatic means. Another advantage of this process is that the marbles with a very small average diameter can be manufactured.

\subsection{Other methods of manufacturing liquid marbles}

Liquid marbles until now have only been manufactured with micro or nanoparticulate materials. While such particles have their advantages such as ease and simplicity of manufacture, they also

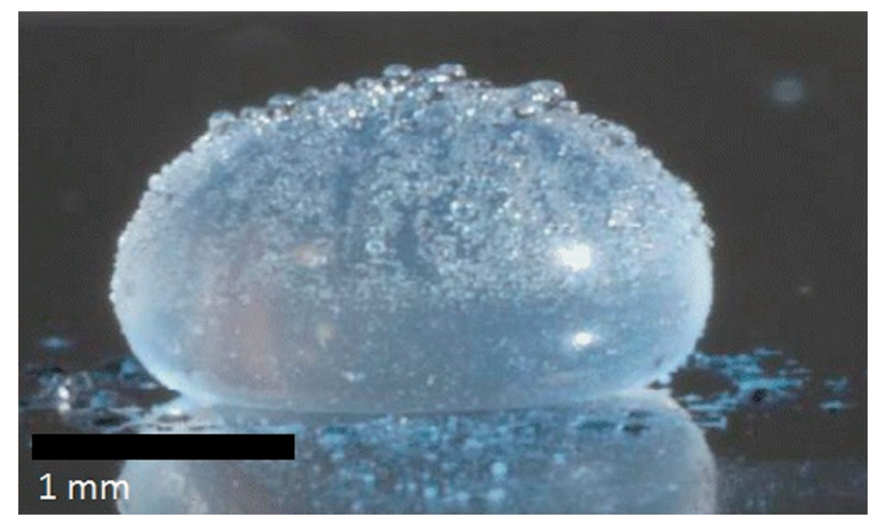

Figure 8. A "sweating" liquid marble. Source: Bhosale \& Panchagnula (2012). Reprinted (adapted) with permission from Bhosale P S and Panchagnula M V 2012 Sweating liquid micro-marbles: dropwise condensation on hydrophobic nanoparticulate materials. Langmuir 28: 14860-. Copyright (2012) American Chemical Society. 
have a few disadvantages. Micro or nanoparticles often do not cover the entire surface of the liquid marble. This can often lead to limitations in robustness or a decrease in the lifetime of the liquid marble. Mele et al (2014) have demonstrated that particulate matter alone does not have a monopoly in the construction of liquid marbles. Uniquely, they have used superhydrophobic nanofibres to coat the droplet of liquid. The nanofibres were made using electrospinning. Fluoroacryllic copolymer and cellulose acetate were used to manufacture hydrophobic nanofibres using this process. The nanofibres form a dense mat which covers the surface of the liquid marble, giving it the desired property. The methodology of manufacture is also simple. The liquid drop is allowed to first fall on the fibrous mat of the superhydrophobic nanofibres. The deformation and kinetic energy of the liquid during impact and rebound extirpates the nanofibres from the mat and assembles them on the liquid-air surface. The energy of the drop is sufficient to carry away a layer of nanofibres with it. Further rolling and manipulation of the drop results in the coverage of the entire drop surface with nanofibres. The entire process takes about five seconds from the initial impact to the final production of the drop. By controlling the physical and chemical compositions of the nanofibres, they were able to create tunable material properties that in turn had an effect of liquid marble properties such as robustness. They were able to create a liquid marble that was stable on a wide range of substrates and was also able to float in water. Evaporation of the liquid was also limited indicating a high fidelity of the coating. The most remarkable testament to the robustness of marbles made in this manner is that they were able to withstand vortex mixing in an oil phase without water losses or rupture even at high speeds. This technique shows good potential to be used in mixing processes.

Apart from nanofibres, other new materials have also been employed to manufacture liquid marbles. Chu et al (2014) have used superhydrophobic carbon-ferric oxide microsheets (graphene) to encapsulate non-volatile liquids and make liquid marbles. Graphene microsheets cover the surface of the marble in a similar fashion to microparticulate matter and exhibit increased robustness and resistance to evaporation. The porous nature of the microparticulate layer is one of the routes for the evaporated vapor to leave the liquid marble. The superhydrophobic microsheets in contrast, do not leave a porous morphology in the liquid marble. The morphology and the relative humidity of the atmosphere greatly influenced both the robustness as well as the rate of evaporation of the liquid marble. Indeed, the evaporation of the encapsulated liquid was greatly reduced under normal conditions when compared to liquid marbles that have been made from superhydrophobic microparticles. The superhydrophobic carbon-ferric oxide microsheets were made by crushing hollow capsules of the same material that had been made in an earlier process. These microsheets have nanoparticles of ferric oxide embedded in carbon. Because of their non-volatile nature, these liquid marbles demonstrated utility in catalysis reactions.

Many of the studies that have been performed so far have used microparticles for the manufacture of the liquid marbles. While liquid marbles can be formed from the microparticulate particles, the liquid marbles thus formed are not sufficiently robust. Using nanoparticulate matter in the place of microparticles can result in increased robustness of liquid marbles under both tensile and compressive loading conditions as studied by Bhosale et al (2008). The resulting liquid marble was not only more robust, but also transparent. Optical transparency implies that these marbles can be interrogated optically during use, a property that becomes useful in biofluidics. They showed that the robustness of these nano-particle covered liquid marbles can be attributed to an elastic film or network of long chain particulate matter that forms on the surface of the marble. The nanoparticles used in this case were fumed silica nanoparticles. For the microparticulate case, the authors used micron-sized polytetrafluoroethylene. Two sets of experiments were used to quantify the robustness of the marble - a tensile load test and an evaporation test. In both 
cases, the nanoparticulate material coated liquid marbles were shown to be the more robust. The increased robustness was postulated to be due to the increased particle-particle interaction in the marbles made from the long chain intertwined nanoparticulate materials.

More interestingly, the authors also performed compressive load testing on the liquid marbles using evaporation. Evaporation of the liquid in the marbles causes a compressive stress to develop on the elastic nanoparticulate film that is stretched over the liquid marble. Eventually, the film is seen to buckle. This buckling is observed sooner in marbles formed from microparticles than in marbles formed from nanoparticles. This is direct proof of robustness under compressive loads as well. Similar tests were also performed by Hu et al (2014), where they demonstrated the utility of highly compressible magnetic liquid marbles made from chainlike superhydrophobic nanoparticles. The marbles thus prepared displayed excellent mechanical robustness and a remarkable self-recovering ability. The marbles demonstrated pronounced elastic behavior ascribed to the entangling of chain-like microstructures on the surface. These chain-like structures form a network that is not easily breached.

As mentioned before, increased marble robustness is a challenge currently impeding industrial applications. As a step in that direction, Fernandes et al (2014) prepared liquid marbles using another novel technique. They encapsulated water droplets using flocculated polymer latexes stabilized with polyionic liquids. They obtained stable latexes composed of spherical polymer particles with sizes ranging from 300 to $700 \mathrm{~nm}$. Gentle shaking of water droplets deposited on the surface of these latexes resulted in stable liquid marbles. The marbles were placed on a water surface to check for stability. The majority of the marbles remained unbroken and stable for more than an hour. Zuber et al (2013) used glass films to coat drops of water to produce (not strictly) liquid marbles. They were shown to be more robust and resistant to evaporation than traditional particle coated liquid marbles. Liu et al (2013) introduced a millifluidic approach in which liquid marbles were continuously generated and collected. They adapted an assembly automation machine called a vibration bowl feeder for this purpose. The maximum rate of fabrication in this process was over 200 marbles per minute. The effects of different operating parameters such as the vibration amplitude, hydrophobic powders, and viscosity of liquid on the properties of the resultant liquid marbles were investigated.

\section{Liquid marbles produced with hydrophilic particles}

The majority of researchers who work with liquid marbles manufacture them with hydrophobic powders. However, liquid marbles manufactured with hydrophilic graphite and carbon black were also reported by Dandan \& Erbil (2009) and Tosun \& Erbil (2009). The possibility of manufacturing of liquid marbles with hydrophilic particles calls for a reasonable thermodynamic explanation. Indeed, it seems that it is thermodynamically favorable for hydrophilic particles to be completely coated by water, thus their location on the surface of liquid marbles appears surprising. Liquid marbles form from hydrophilic materials as a result of the shape of particulate matter. The shape (for example, a sphere or a cone) remains trapped in a metastable state where increased wetting of the particle is energetically expensive. This also causes the marble to remain metastable. In addition, it was suggested that hydrophilic particles form aggregates which trap air in them as depicted in figure 9. The air cushions shown in figure 9 promote the Cassie \& Baxter (1944) wetting regime leading to their hydrophobicity. However, this hypothesis calls for direct experimental observation. 


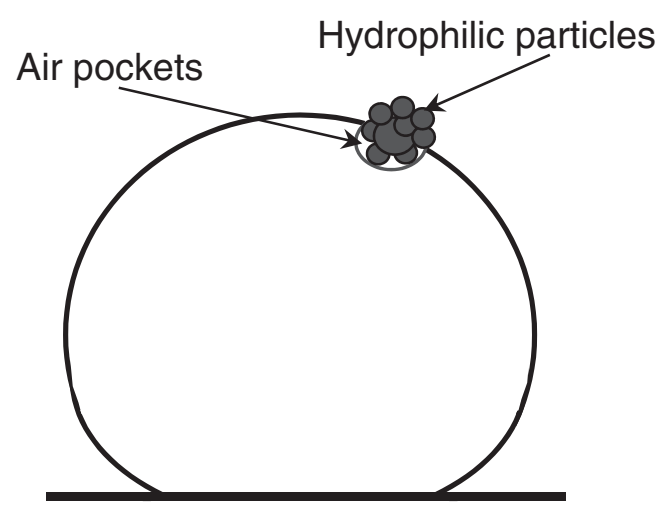

Figure 9. Cassie Baxter wetting at the interface.

\section{Applications of liquid marbles}

Liquid marbles promise a variety of applications in various areas such as microfluidics, micro transport of liquids, catalysis and micro reactions. A few promising ones are discussed hereunder.

\subsection{Actuation of liquid marbles with electric and magnetic fields}

It has been shown by various groups, that liquid marbles could be actuated by electric and magnetic fields, which make microfluidics applications of liquid marbles possible. Let us begin with magnetic actuation of liquid marbles. It was shown by Bormashenko et al (2008) that ferrofluidic liquid marbles that are accelerated by a magnetic field reached velocities as high as 25 $\mathrm{cm} / \mathrm{s}$. Zhao et al (2012) reported liquid marbles coated with highly hydrophobic $\mathrm{Fe}_{3} \mathrm{O}_{4}$ particles. These magnetic nanoparticles in this case played a dual role, as both a force mediator and the hydrophobic coating. Under the influence of a magnetic field, the magnetic liquid marbles were not only manipulated in both two-dimensional and three-dimensional environments but were also made to open and close reversibly, depending upon the intensity of the magnetic field applied. The fluid can be easily removed or refilled into such marbles in the open state. In addition, two opened marbles can be made to merge into a larger marble. This level of micro-manipulation of the marble as well as its liquid content presents a hitherto unavailable degree of freedom to a microfluidic system designer. Further, Zhao et al (2012) have also reported the effective use of magnetically opened marbles for optical probing which can be used for diagnostics.

Electric fields also have been implemented to manipulate the liquid marbles by Aussillous \& Quéré (2006) and McHale et al (2006). The dimensionless constants $\xi_{1}, \xi_{2}$ describing the sensitivity of the marble to the electric field could be introduced as suggested by Bormashenko et al (2012). The constant $\xi_{1}$ describes the interrelation of the electrically induced effects and gravity:

$$
\xi_{1}=\frac{\varepsilon \varepsilon_{o}}{\rho g R} E^{2},
$$

where $\varepsilon$ and $\rho$ are the dielectric constant and density of the marble respectively. The constant $\xi_{2}$ describes the interrelation of electrically induced and the effective surface tension:

$$
\xi_{2}=\frac{\varepsilon \varepsilon_{o} R}{\gamma_{e f f}} E^{2},
$$


when $\xi_{1} \cong \xi_{2} \cong 1$, the marble becomes sensitive to electric field. Substituting $\epsilon=2.1 ; \rho \cong$ $1000 \frac{\mathrm{kg}}{\mathrm{m}^{3}} ; \gamma_{\text {eff }} \cong 50 \frac{\mathrm{mj}}{\mathrm{m}^{2}}$ yields an estimate of the required electric field, $E^{*}$ for actuation without rupture. For electrical actuation of liquid marbles, an estimate of $E^{*} \cong 10^{6} \frac{\mathrm{V}}{\mathrm{m}}$ has been shown to be in agreement with the experimental findings. Thus, relatively large voltages are still necessary for the electrical actuation of liquid marbles. Methods allowing low-voltage actuation of liquid marbles need to be researched.

\subsection{Use of liquid-marbles as micro-reactors}

The permeability of the liquid marble shell, thanks to the porous nature of the microparticulate covering on the liquid marble surface, has led to a number of interesting applications in gas sensing as shown by Nguyen et al (2010) and Tian et al (2010). pH sensing is an important application in the area of liquid marbles as demonstrated by Fujii et al (2010). Pollution sensing of water has also been an emerging area of research as shown by Bormashenko \& Musin (2009). Some of the recent applications are summarized below.

Miao et al (2014) fabricated silver nanowire-based liquid marbles to act as miniature reactors. These micro reactors demonstrated high efficiency, support free and rate controllable reactions with a catalytic efficiency of nearly $100 \%$. These liquid marbles were shown to be effective in situations where toxic or hazardous materials were used and small volume preliminary reactions are preferred. Liquid marbles have found application as miniature reactors due to the reduced usage of solvents and reagents, confined micro-environmental and precisely controllable reaction conditions. The properties of liquid marbles are unique and are useful for minimizing the dangers of highly toxic reagents in hazardous reactions.

Perfluorodecanethiol-grafted Ag nanowires were used as the superhydrophobic material that covered the water droplets. When water droplets are dispensed on a bed of perfluorodecanethiolgrafted Ag nanowires, spherical water liquid marbles were formed. The silver nanowires were observed to spontaneously assemble at the interface of the air/water. The length of the pulverized $\mathrm{Ag}$ nanowires required for the formation of uniform and robust catalytic liquid marbles was determined. The shorter Ag nanowires were able to provide a porous Ag nanowire network with a very large catalytic surface area while the longer Ag nanowires provide stability. Thus, a porous shell was more desirable and achieved. The catalytic reduction of encapsulated aqueous methylene blue in the presence of sodium borohydride $\left(\mathrm{NaBH}_{4}\right)$ was studied to validate the use of these liquid marbles as catalytic reactors. The liquid marble was ruptured at predetermined timings and the progress of the reaction was monitored. The Ag nanowires imparted a high ratio of surface area to volume and the porous network structure allowed the exposure of a large number of sites for efficient catalytic reaction.

Bioreactors are an emerging area in which liquid marbles have been utilized recently. Sarvi et al (2014) prepared a liquid marble micro-bioreactor by placing a drop of murine embryonic stem cell suspension onto a bed of polytetrafluoroethylene (PTFE) particles. The stem cells aggregated to form embryoid bodies with a relatively uniform size and shape in a liquid marble. Some of the suspended embryoid bodies exhibited spontaneous contraction. All of this indicated that the liquid marble provided a suitable environment to induce embryoid body formation. Some of the embryoid bodies were subsequently plated onto gelatin-coated tissue culture dishes. They demonstrated that the liquid-marble technique was an easily employed, cost-effective, and efficient approach to generate embryoid bodies and facilitating their cardio genesis. 
Tan et al (2014) applied the concept of liquid marbles to photoreceptivity and similar sensing mechanisms. They manufactured liquid marbles by coating water with titanium dioxide nanoparticles, which are known to display photoreceptive wettability. Water liquid marbles stabilized by these photoresponsive titanium dioxide particles were found to be stable when shielded from ultraviolet radiation. However, they quickly collapsed after being irradiated with ultraviolet light. Titanium dioxide is a relatively cheap and inexpensive material, and the manufacture of which is also simple when compared to some of the other superhydrophobic particles that have been used in the manufacture of liquid marbles. Therefore, actuating the contents of a marble using ultraviolet light exposure presents itself useful to several applications.

Sensing applications hold the most promise among liquid marbles thanks to the minute quantities of fluid involved as well as the porous nature of the microparticulate shell that covers the liquid. Tian et al (2010) have shown that the porous and superhydrophobic shell of a liquid marble restricts the contact between the liquid and the substrate, but allows the transport of gas. Therefore, liquid marbles can be used to sense gases. They performed qualitative experiments with ammonia and various liquid marbles made from different liquids such as water and phenolphthalein and demonstrated that the color change that accompanied the introduction of the ammonia gas was rapid and immediate. The rapid color change of the indicator liquids was confirmation that the porous liquid marble shell allows the passage of gas molecules through the shell. The degree to which the marble changed color was quantified as an indication of the amount of ammonia present in the solution. This application lends itself to many fluid-gas indicator combinations.

\section{Future research directions}

Liquid marbles present an alternative to classical, roughness-inspired superhydrophobicity. They involve exciting new phenomenon and hold great promise and potential for many applications in a wide variety of fields. The applications range from chemistry and biosciences to physics and allied sciences. Great strides have been made in understanding the materials that can be employed as the encapsulating materials and the limitations in each case. Research has also been focused on methods of actuation as well as understanding the marbles' response to such fields. However, two important fields of study related to liquid marbles require critical attention before these objects can see industrial applications can be realized. Studies can also be performed in analyzing the average distribution of the solid particles over the surface and their effect on liquid marble properties.

Listing potential applications in their original paper, Aussillous \& Quéré (2001) say, "Liquid marbles thus seem to be an interesting solution to the problem of driving small amounts of liquid on solid (or even liquid) substrates." They go onto add, "We are at present examining the notable robustness of these marbles, which are able to resist a series of shocks." Thirteen years later, the application they proposed remains unrealized and the physical phenomenon that they identified as a performance limiter remains under-researched. One possible reason for this lacuna is that the community has focused mostly on the creation and characterization of single liquid marbles. We herein list a few topics of possible research to motivate researchers.

(a) Robustness of liquid marbles is a performance limiter, in applications where unintended rupture is not desired. Rupture due to collision with a solid surface has been well understood. However, rupture due to marble-marble collision, especially in a dynamical setting (while rolling, for example) remains a topic requiring attention. One of the factors determining the 
robustness of liquid marbles is the degree of spatial uniformity of the particulate sheath. Spatial inhomogeneity determines regions where the marble is vulnerable to rupture. To the best of our knowledge, this aspect of marble robustness has not been studied experimentally.

(b) Dynamics of large numbers of liquid marbles in a rolling and colliding needs to be understood in order to realize applications in large scale liquid transport using encapsulation. It is important to realize that a simple superposition of single marble or even two-marble dynamics may not be sufficient in this regard, as the damage caused by successive two-marble collisions may accumulate and cause rupture.

(c) The particulate sheath that protects the marble from rupture has been shown to manifest various phases, as described earlier. During rolling and transport however, this particulate sheath may undergo phase changes, as the particles are generally mobile at the liquid-vapor interface. It would be desirable to understand these transitions, especially to engineer such transition where damage (say due to two-marble collisions) can be healed. Such self-healing systems have been proposed in other areas and seem to hold general promise.

(d) With regard to applications, research at the device level is lacking conspicuously. Device level research and development is important, as it provides both motivation and focus to the more fundamental questions that need to be answered. For example, bulk transport of high viscosity liquids has been proposed, but no device level research has been reported on this problem.

(e) The transport of liquid marbles in confined channels has not been studied in the literature. An example application where this information is useful occurs in microfluidic channels. While liquids encapsulated in a particulate sheath provide advantages in terms of drag reduction during transport, the robustness of the marble in such a setting needs to be investigated.

\section{References}

Arbatan T and Shen W 2010 A preliminary study on gas transportation through porous superhydrophobic surfaces and its application in gas sensing. Chemeca 2010: Engineering at the Edge; 26-29 September 2010, Hilton Adelaide, South Australia 3527

Aussillous P and Quéré D 2001 Liquid marbles. Nature 411: 924-927

Aussillous P and Quéré D 2006 Properties of liquid marbles. Proc. R. Soc. A: Math. Phys. Eng. Sci. 462: 973-999

Beysens D 2006 Dew nucleation and growth. Comptes Rendus Physique 7: 1082-1100

Bhosale P S and Panchagnula M V 2010 On synthesizing solid polyelectrolyte microspheres from evaporating liquid marbles. Langmuir 26: 10745-10749

Bhosale P S and Panchagnula M V 2012 Sweating liquid micro-marbles: dropwise condensation on hydrophobic nanoparticulate materials. Langmuir 28: 14860-14866

Bhosale P S, Panchagnula M V and Stretz H A 2008 Mechanically robust nanoparticle stabilized transparent liquid marbles. Appl. Phys. Lett. 93: 034109

Biance A-L, Clanet C and Quéré D 2003 Leidenfrost drops. Physics of Fluids (1994-present) 15: 16321637

Binks B P and Murakami R 2006 Phase inversion of particle-stabilized materials from foams to dry water. Nat. Mater. 5: 865-869

Bormashenko E, Bormashenko Y, Musin A and Barkay Z 2009a On the mechanism of floating and sliding of liquid marbles. ChemPhysChem 10: 654-656

Bormashenko E and Musin A 2009 Revealing of water surface pollution with liquid marbles. Appl. Surface Sci. 255: 6429-6431 
Bormashenko E, Musin A, Whyman G, Barkay Z, Starostin A, Valtsifer V and Strelnikov V 2013 Revisiting the surface tension of liquid marbles: Measurement of the effective surface tension of liquid marbles with the pendant marble method. Colloids Surfaces A: Physicochem. Eng. Aspects 425: 15-23

Bormashenko E, Pogreb R, Balter R, Gendelman O and Aurbach D 2012 Composite non-stick droplets and their actuation with electric field. Appl. Phys. Lett. 100: 151601

Bormashenko E, Pogreb R, Bormashenko Y, Musin A and Stein T 2008 New investigations on ferrofluidics: ferrofluidic marbles and magnetic-field-driven drops on superhydrophobic surfaces. Langmuir 24: 12119-12122

Bormashenko E, Pogreb R, Whyman G and Musin A 2009b Surface tension of liquid marbles. Colloids Surfaces A: Physicochem. Eng. Aspects 351: 78-82

Bormashenko E, Pogreb R, Whyman G, Musin A, Bormashenko Y and Barkay Z 2009c Shape, vibrations, and effective surface tension of water marbles. Langmuir 25: 1893-1896

Cassie A B D and Baxter S 1944 Wettability of porous surfaces. Transact. Faraday Soc. 40: 546-551

Celestini F and Kofman R 2006 Vibration of submillimeter-size supported droplets. Phys. Rev. E 73: 041602

Chu Y, Wang Z and Pan Q 2014 Constructing robust liquid marbles for miniaturized synthesis of graphene/Ag nanocomposite. ACS Appl. Mater. Interfaces 6: 8378-8386

Dandan M and Erbil H Y 2009 Evaporation rate of graphite liquid marbles: comparison with water droplets. Langmuir 25: 8362-8367

de Gennes P G, Brochard-Wyart F and Quéré D 2003 Capillarity and wetting phenomena. Berlin: Springer 226-235

Fernandes A M, Gracia R, Leal G P, Paulis M and Mecerreyes D 2014 Simple route to prepare stable liquidmarbles using poly(ionic liquid)s. Polymer 55: 16, 3397-3403

Fujii S, Kameyama S, Armes S P, Dupin D, Suzaki M and Nakamura Y 2010 pH-responsive liquid marbles stabilized with poly(2-vinylpyridine) particles. Soft Matter 6: 635-640

Hu Y, Jiang H, Liu J, Li Y, Hou X and Li C 2014 Highly compressible magnetic liquid marbles assembled from hydrophobic magnetic chain-like nanoparticles. RSC Adv 4: 3162-3164

Liu Y, Hugentobler C P and Shum H C 2013 A millifluidic approach for continuous generation of liquid marbles. J. Colloid Sci. Biotechnol. 2: 350-354

Mahadevan L and Pomeau Y 1999 Rolling droplets. Phys. Fluids (1994-present) 11: 2449-2453

McHale G, Herbertson D L, Elliott S J, Shirtcliffe N J and Newton M I 2006 Electrowetting of nonwetting liquids and liquid marbles. Langmuir 23: 918-924

Mele E, Bayer I S, Nanni G, Heredia-Guerrero J A, Ruffilli R, Ayadi F, Marini L, Cingolani R and Athanassiou A 2014 Biomimetic approach for liquid encapsulation with nanofibrillar cloaks. Langmuir 30: 28962902

Miao Y-E, Lee H K, Chew W S, Phang I Y, Liu T and Ling X Y 2014 Catalytic liquid marbles: Ag nanowire-based miniature reactors for highly efficient degradation of methylene blue. Chem. Commun. 50: 5923-5926

Monteux C, Kirkwood J, Xu H, Jung E and Fuller G G 2007 Determining the mechanical response of particle-laden fluid interfaces using surface pressure isotherms and bulk pressure measurements of droplets. Phys. Chem. Chem. Phys. 9: 6344-6350

Nguyen T H, Eshtiaghi N, Hapgood K P and Shen W 2010 An analysis of the thermodynamic conditions for solid powder particles spreading over liquid surface. Powder Technol 201: 306-310

Nosonovsky M and Bhushan B 2008 Biologically inspired surfaces: broadening the scope of roughness. Adv. Funct. Mater. 18: 843-855

Nosonovsky M and Bormashenko E 2009 Lotus effect: superhydrophobicity and self-cleaning. Functional properties of bio-inspired surfaces $43-78$

Quéré D and Reyssat M 2008 Non-adhesive lotus and other hydrophobic materials. Philos. Transact. R. Soc. A: Math. Phys. Eng. Sci. 366: 1539-1556

Richard D and Quéré D 1999 Viscous drops rolling on a tilted non-wettable solid. Europhys. Lett. 48: 286 
Sarvi F, Jain K, Arbatan T, Verma P J, Hourigan K, Thompson M C, Shen W and Chan P P Y 2014 Cardiogenesis of embryonic stem cells with liquid marble micro-bioreactor. Adv. Healthcare Mater. n/a$\mathrm{n} / \mathrm{a}$

Tan T T Y, Ahsan A, Reithofer M R, Tay S W, Tan S Y, Hor T S A, Chin J M, Chew B K J and Wang X 2014 Photoresponsive liquid marbles and dry water. Langmuir 30: 3448-3454

Tian J, Arbatan T, Li X and Shen W 2010 Liquid marble for gas sensing. Chem. Commun. 46: 4734-4736

Tosun A and Erbil H Y 2009 Evaporation rate of PTFE liquid marbles. Appl. Surface Sci. 256: 1278-1283

Vella D, Aussillous P and Mahadevan L 2004 Elasticity of an interfacial particle raft. Europhys. Lett. 68: 212-218

Venkateswara Rao A, Kulkarni M M and Bhagat S D 2005 Transport of liquids using superhydrophobic aerogels. J. Colloid Interface Sci. 285: 413-418

Whyman G and Bormashenko E 2009 Oblate spheroid model for calculation of the shape and contact angles of heavy droplets. J. Colloid Interface Sci. 331: 174-177

Zhao Y, Xu Z, Parhizkar M, Fang J, Wang X and Lin T 2012 Magnetic liquid marbles, their manipulation and application in optical probing. Microfluidics Nanofluidics 13: 555-564

Zuber K, Evans D and Murphy P 2013 Nanoporous glass films on liquids. ACS Appl. Mater. Interfaces 6: $507-512$ 